\title{
An extended singular spectrum transformation (SST) for the investigation of Kenyan precipitation data
}

\author{
N. Itoh ${ }^{1}$ and N. Marwan ${ }^{2}$ \\ ${ }^{1}$ University of Potsdam Interdisciplinary Center for Dynamics of Complex Systems (DYCOS), Campus Golm, \\ Building 14 Karl-Liebknecht-Str. 24, 14476, Potsdam, Germany \\ ${ }^{2}$ Potsdam-Institut für Klimafolgenforschung (PIK) e.V., Telegrafenberg A31, 14473, Potsdam, Germany
}

Correspondence to: N. Itoh (naoki.itoh@gmail.com)

Received: 1 March 2013 - Revised: 15 May 2013 - Accepted: 16 May 2013 - Published: 12 July 2013

\begin{abstract}
In this paper a change-point detection method is proposed by extending the singular spectrum transformation (SST) developed as one of the capabilities of singular spectrum analysis (SSA). The method uncovers change points related with trends and periodicities. The potential of the proposed method is demonstrated by analysing simple model time series including linear functions and sine functions as well as real world data (precipitation data in Kenya). A statistical test of the results is proposed based on a Monte Carlo simulation with surrogate methods. As a result, the successful estimation of change points as inherent properties in the representative time series of both trend and harmonics is shown. With regards to the application, we find change points in the precipitation data of Kenyan towns (Nakuru, Naivasha, Narok, and Kisumu) which coincide with the variability of the Indian Ocean Dipole (IOD) suggesting its impact of extreme climate in East Africa.
\end{abstract}

\section{Introduction}

Many processes in nature, engineering, or life can be studied by measuring quantities and analysing such time series (Bishop, 2007; Brockwell and Davis, 1991, 2002). Typically such a purpose is achieved by using statistical tools. Following the development of computer performance, in particular, it allows us to analyse a large amount of data for a number of different parameters. The climate data analysis discussed in this paper is one typical case.

As one of the most typical climate phenomena, El Niño and La Niña occurring in the Pacific Ocean by the dynamics of the atmosphere-ocean interaction are known, which are mostly studied as a problem of El Niño/Southern Oscillation (ENSO). Recently, a coupled ocean-atmosphere phenomenon called the Indian Ocean Dipole (IOD) similar to the ENSO has been exposed (Saji et al., 1999). This phenomenon is apparently as important as the intertropical convergence zone (ITCZ) for the tropical and sub-tropical regions. In particular, it is considered that IOD is influencing rainfall in East Africa. However Saji et al. mentioned that the IOD events occur independently of the ENSO events which are observed in the Pacific region expressed by the NINO3 index $\left(5^{\circ} \mathrm{N}-5^{\circ} \mathrm{S}, 150-90^{\circ} \mathrm{W}\right)$. Figure 1 shows the comparison between the monthly behaviours of IOD and NINO3 time series (Fig. 1a) and the correlation coefficients of the past ten years at every time point (Fig. 1b). From the figure, the positive IOD in the Indian Ocean and the El Niño in the Pacific Ocean often occur simultaneously, e.g. as in 1972 and 1997. The reason may be that the sea surface temperatures in the western Indian Ocean and in the eastern Pacific Ocean are higher than the opposite sides of these oceans that are located around Indonesia. On the other hand, the positive IODs in 1961, 1967, and 1994 occurred with the ENSO indices that express no El Niño, a La Niña, and a weak El Niño, respectively, in which heavy rainfalls were provided in East Africa in 1961 (Flohn, 1987). Saji et al. (1999) mentioned, therefore, that the correlation between the IOD and ENSO is weak. In this study, as shown by the red rectangles in Fig. 1, we focus on the significant uncorrelation time in the 1960s. Since it is different from the correlation at other times, the motivation in this study is to consider the IOD as a complementary index of the climate variability in East Africa in addition to the ENSO. 
The IOD shows that anomalies occurring between the eastern and western equatorial Indian Oceans (Fig. 2) can be decomposed into a positive and a negative modes. When the Indian Ocean is in the normal state (i.e. the index is zero) the sea surface temperature is relatively high in the east and low in the west. The dipole mode depends on the strength of the south-east trade winds. The positive dipole mode is illustrated in Fig. 2a: if the wind on the Indian Ocean grows relatively strongly, the hot sea water in the eastern side moves to the western side and thus the sea surface temperature in the western side increases according to this effect and the sea surface temperature in the eastern side will drop by the upwelling cold water coming from the deep sea. At this time, since a atmospheric convective activity moves to the west, then floods are often caused in East Africa as well as Indonesia is besieged by droughts. The negative dipole mode is illustrated in Fig. 2b: if the wind relatively weakens, the normal state will furthermore grow strongly, i.e. the sea surface temperature in the east is warmer and the sea surface temperature in the west is colder. At this time, in contrast to the positive dipole mode, the convective activity happens in the east, which causes droughts in East Africa and floods in Indonesia. According to recent reports (Ashok et al., 2001; Behera et al., 2005; Saji et al., 1999), such a "teeter-totter" phenomenon alternately causes droughts/floods in East Africa on the west side and in Indonesia on the east side of the Indian Ocean. According to Nakamura et al. (2009), the periodicity of IOD in recent decades was quasi-biennial (1.5$3 \mathrm{yr}$ ), which is shorter than at the beginning of the 20th century. In the article, it was suggested that tropical convections were encouraged due to a warming of the sea surface temperature in the western Indian Ocean. Then an occurrence of the positive IOD event, which brings in heavy rainfall in East Africa, became more frequent. Furthermore, Nakamura et al. (2009) mentioned that the ENSO effect weakened by the warming tendency in the western Indian Ocean and alternatively the IOD effect increased over the Indian Ocean since 1960. Therefore, we focus on the 1960s and aim at the characterisation of the change in the IOD dynamics by using a change-point detection approach.

In this study, the monthly precipitations in Nakuru $\left(0^{\circ} 17^{\prime} \mathrm{S}, 36^{\circ} 4^{\prime} \mathrm{E}\right)$, Naivasha $\left(0^{\circ} 43^{\prime} \mathrm{S}, 36^{\circ} 25^{\prime} \mathrm{E}\right)$, Narok $\left(1^{\circ} 5^{\prime} \mathrm{S}, 35^{\circ} 52^{\prime} \mathrm{E}\right)$, and Kisumu $\left(0^{\circ} 6^{\prime} \mathrm{S}, 34^{\circ} 45^{\prime} \mathrm{E}\right)$ provided from the GHCN v2 database (http://www.ncdc.noaa.gov/) will be compared with the IOD. Figure 3 shows the locations of these stations. This region is located around the Equator. Therefore since it is strongly affected according to a seasonal movement of the ITCZ formed by a convergence of Hadley circulation, there are two rainy seasons in spring (a long term) and autumn (a short term). The obtained data have different time length. However, as shown in Fig. 4 the data used in this study are overlapping at all the stations in the time region between January 1950 and December 1985 (for $36 \mathrm{yr}$ ) corresponding to 432 data points.
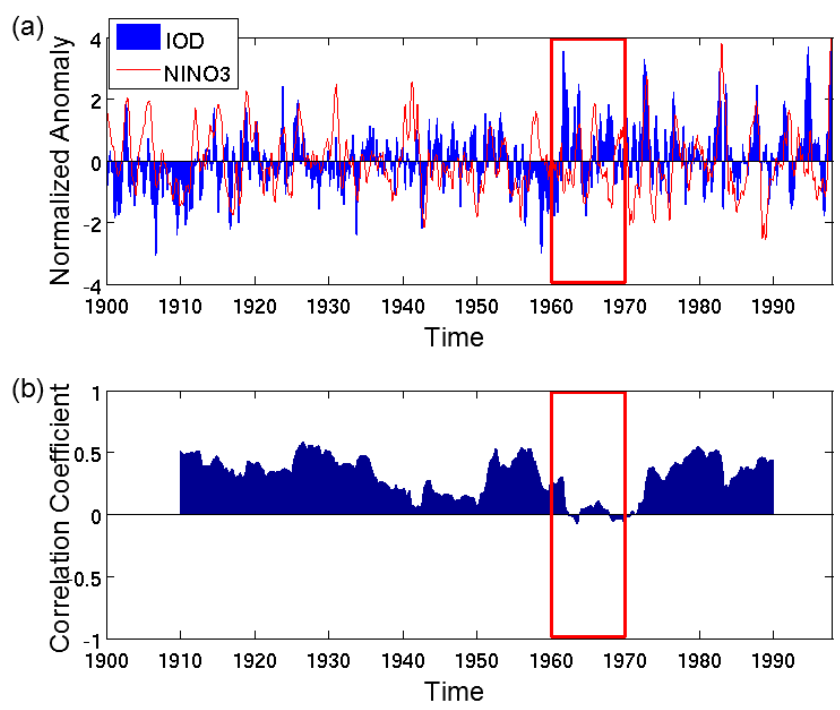

Fig. 1. (a) Normalised time series (mean zero and variance one) of IOD (from Japan Agency for Marine Earth Science and Technology; http://www.jamstec.go.jp) and ENSO (from KNMI Climate Explorer; http://climexp.knmi.nl) from 1900 to 1997. (b) Running correlation coefficients between IOD and ENSO for the past $10 \mathrm{yr}$ at all the time points. Both time series have been smoothed by using a 3 -month moving average. The ENSO is represented by NINO3 sea temperature anomalies. By the red rectangles the 1960s is emphasised, where the IOD index obviously exhibits a behaviour different from that of ENSO in the 1960s and then the correlation between them is low $(<0.1)$ during this time.

The SSA has provided a number of typical achievements in many scientific fields, especially, it can be used for solving the following problems: finding trends of different resolution, smoothing, extraction of seasonality components, simultaneous extraction of cycles with small and large periods, extraction of periodicities with varying amplitudes, simultaneous extraction of complex trends and periodicities, finding structures in short time series, and even change-point detection (Hassani, 2007). The basic concept of SSA derives from the principal component analysis (PCA) and the singular value decomposition (SVD) (Björnsson and Venegas, 1997). A comprehensive discussion is described in the papers and books by Elsner and Tsonis (1996); Vautard et al. (1992); Ghil et al. (2002); Golyandina et al. (2001).

Change-point detection in time series can be performed by different techniques. For example, Mudelsee (2000) suggested detecting change points in palaeoclimate time series by using a regression model called RAMPFIT. As a more recent technique, a change-point detection by using a Bayesian approach was applied to the annual flow volume of the Nile River (Schütz and Holschneider, 2011). These methods assume a change in the linear behaviour (trends) and require a parametric or a distribution fit. In contrast, the changepoint technique based on SSA is nonparametric and it is not necessary to estimate a statistical distribution or to fit a 
(a)

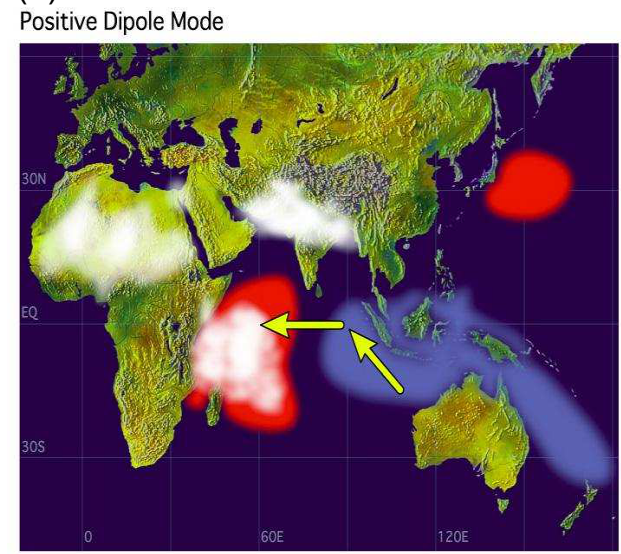

(b)

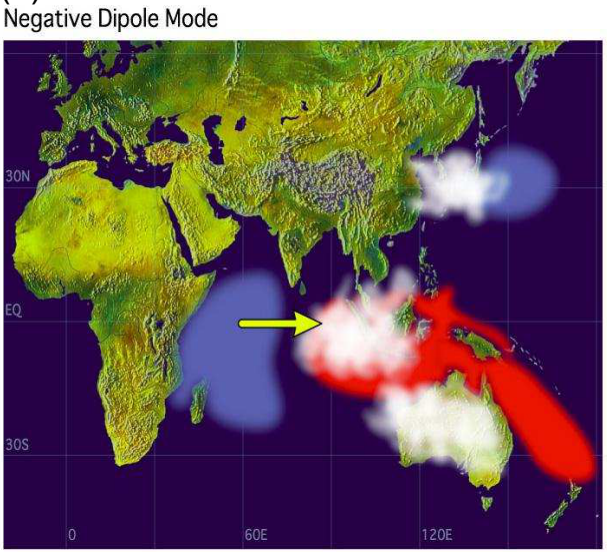

Fig. 2. Schematic of (a) positive IOD mode, (b) negative IOD mode (from Japan Agency for Marine Earth Science and Technology, http://www.jamstec.go.jp).

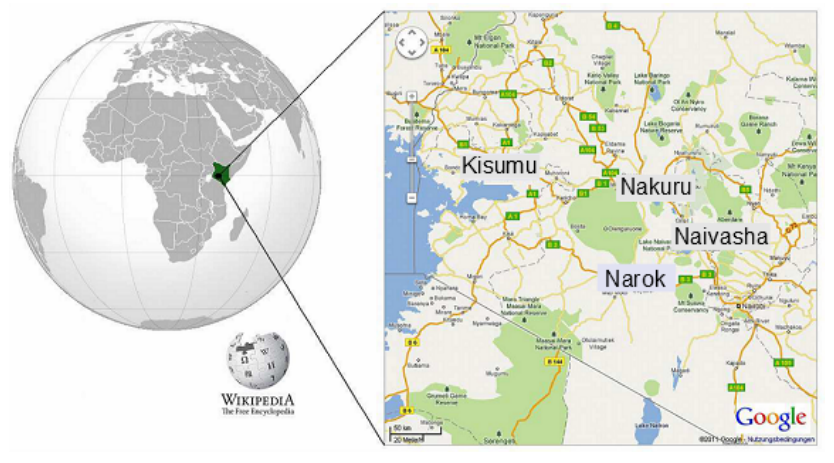

Fig. 3. Location of the used weather stations in Kenya. Nakuru $\left(0^{\circ} 17^{\prime} \mathrm{S}, 36^{\circ} 4^{\prime} \mathrm{E}\right)$, Naivasha $\left(0^{\circ} 43^{\prime} \mathrm{S}, 36^{\circ} 25^{\prime} \mathrm{E}\right)$, Narok $\left(1^{\circ} 5^{\prime} \mathrm{S}\right.$, $\left.35^{\circ} 52^{\prime} \mathrm{E}\right)$, and Kisumu $\left(0^{\circ} 6^{\prime} \mathrm{S}, 34^{\circ} 45^{\prime} \mathrm{E}\right)$.

linear trend (Idé and Inoue, 2004, 2005; Idé, 2006; Itoh and Kurths, 2010; Mohammad and Nishida, 2009; Moskvina and Zhigljavsky, 2003). This is an advantage because it is difficult to reliably estimate parameters and distributions for short observations.

A change point is generally defined as any time point in the evolution of a system in which an abrupt structural and dynamical change occurs. Such a change may appear in time series as discontinuities, gaps or changes in periodicities, amplitude or variance. The basic motivations for this investigation are explained by the following assumptions: the properties or parameters describing the data are either constant or slowly time-varying. In practical problems, abrupt changes occurring at certain times can help in modelling dynamical processes (Basseville and Nikiforov, 1993). Focusing on a climate application in this study, change-point detection
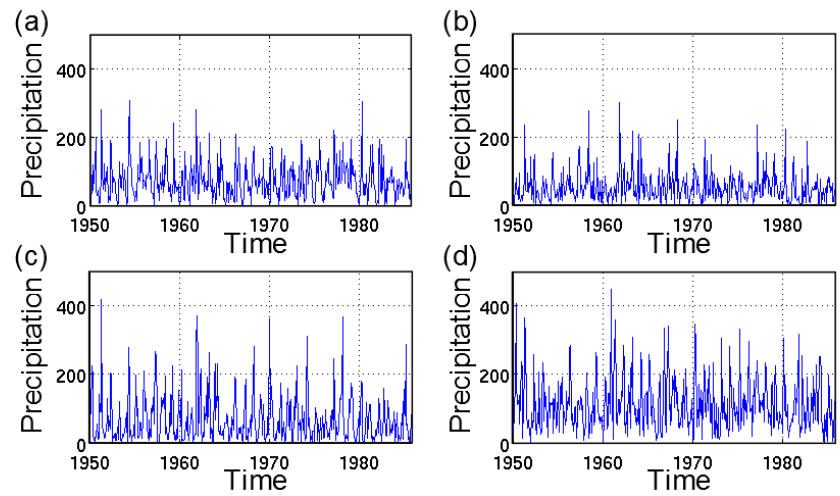

Fig. 4. Observations of monthly precipitation between 1950 and 1985. (a) Nakuru, (b) Naivasha, (c) Narok, and (d) Kisumu.

may provide basic insights into abrupt climate changes of a trend with a long-term variability but also seasonal cycle or quasiperiodicity. If such a change point can clearly be identified in a time series the result will suggest that there exist substantially different properties or structures in the climate sense before and after the time instant.

In this study the SSA-based change-point detection proposed by Idé and Inoue (2004), has been applied, which is called singular spectrum transformation (SST). However, the conventional SST has considered only change points for the most dominant component of data corresponding to a change in a trend. Since the SSA can originally reveal several representative orthogonal components from data we will extend the conventional SST to detect the change points also for further components. In the next section we explain the procedures of the SSA as well the SST and then propose the extended version of SST. 


\section{Method}

\subsection{Singular spectrum analysis (SSA)}

The SSA aims to extract spectral information from time series. As described in Sect. 1, the change-point detection is one of the capabilities of the SSA. Basically, this is achieved by mathematically decomposing and reconstructing a time series (Elsner and Tsonis, 1996; Golyandina et al., 2001).

The first step is an embedding of a single time series $\boldsymbol{Y}=\left(y_{1}, \cdots, y_{N}\right)$ into a multidimensional vector space, what can be achieved by transforming the time series into the following trajectory matrix of size $\mathbf{K} \times L,(K=N-L+1)$ :

$\boldsymbol{X}=\left[\boldsymbol{X}_{1}: \cdots: \boldsymbol{X}_{K}\right]=\left(x_{i j}\right)_{i, j=1}^{L, K}$,

where $\boldsymbol{X}_{i}=\left(y_{i}, \ldots, y_{i+L-1}\right)^{T},(1 \leq i \leq K)$. The matrix is expressed as a Hankel matrix, in which elements on the anti diagonals are equal (Phillips, 1971). The dimension or window length $L$ is directly related to the fineness of the decomposition of the original time series in order to characterise essential aspects of the data.

In the second step, the trajectory matrix is decomposed by the SVD in the following form:

$\mathbf{X}=\mathbf{U} \mathbf{S} \mathbf{V}^{T}$,

where two unitary matrices of singular vectors, $\mathbf{U}$ and $\mathbf{V}$ consist of orthonormal vectors regarded as basis vectors of the trajectory matrix and $\mathbf{S}$ means a matrix of singular values including nonnegative real numbers on the main diagonal and zero in the remaining positions. Then the trajectory matrix is represented by rank-one biorthogonal elementary matrices calculated according to the singular vectors and the singular values. That is, the trajectory matrix can be written as

$\mathbf{X}=\mathbf{X}_{1}+\cdots+\mathbf{X}_{d}, \quad \mathbf{X}_{j}=\sqrt{\lambda_{j}} \boldsymbol{U}_{j} \boldsymbol{V}_{j}^{T}=\left(x_{i k}^{[j]}\right)_{i, k=1}^{L, K}$,

where $j=1, \ldots, d(\leq L)$ because the singular values $\sqrt{\lambda_{j}}$ are arranged in decreasing order of magnitudes as $d=$ $\max \left\{j\right.$, such that $\left.\lambda_{j}>0\right\}=\operatorname{rank} \mathbf{X}$. Note that these singular vectors, $\boldsymbol{U}$ and $\boldsymbol{V}$ represent the orthogonal decomposed properties of the time series and the singular values, $S$ just quantifies the magnitudes of the components. The collection $\left(\lambda_{j}, \boldsymbol{U}_{j}, \boldsymbol{V}_{j}\right)$ is called the $j$-th "eigentriple" of the trajectory matrix

The third step is the reconstruction of new time series from the elementary matrix. By taking the feature of the Hankel matrix it is possible to suppose that the elements on the diagonal from a left bottom to a right upper (i.e. a secondary diagonal line) in the matrix derive from the event occurred at the same time. A mean value of such elements can, therefore, be considered as a data point decomposed from the original time series. This technique can be applied to all elementary matrices and then it is called diagonal averaging reconstruc- tion. The calculation can be expressed as follows:

$$
\hat{y}_{n}^{[j]}= \begin{cases}\frac{1}{n} \sum_{m=1}^{n} x_{m,(n-m+1)}^{[j]} & (1 \leq n<L) \\ \frac{1}{L} \sum_{m=1}^{L} x_{m,(n-m+1)}^{[j]} & (L \leq n<K), \\ \frac{1}{N-n} \sum_{m=n-K+2}^{N-K+1} x_{m,(n-m+1)}^{[j]} & (K \leq n<N)\end{cases}
$$

where $\hat{y}_{n}^{[j]}$ means an element computed from the $j$-th eigentriple. Then its reconstructed time series is defined as $\hat{\boldsymbol{Y}}^{[j]}=$ $\left(\hat{y}_{1}^{[j]}, \cdots, \hat{y}_{N}^{[j]}\right)$. Note that these reconstructed time series are orthogonal with each other. Thus the original time series can be expressed as the sum of these time series, that is, $\boldsymbol{Y} \approx \hat{\boldsymbol{Y}}^{[1]}+\ldots+\hat{\boldsymbol{Y}}^{[d]}$.

In the fourth step, to group these components into several representative time series, the singular values and weighted $(w)$ correlation may provide some hints for grouping. The singular values have already been obtained by SVD. The $w$ correlation is calculated as follows:

$\boldsymbol{\rho}_{a b}^{(w)}=\frac{\left(\hat{\boldsymbol{Y}}^{[a]}, \hat{\boldsymbol{Y}}^{[b]}\right)_{w}}{\left\|\hat{\boldsymbol{Y}}^{[a]}\right\|_{w}\left\|\hat{\boldsymbol{Y}}^{[b]}\right\|_{w}},\left\|\hat{\boldsymbol{Y}}^{[\times]}\right\|_{w}=\sqrt{\left(\hat{\boldsymbol{Y}}^{[\times]}, \hat{\boldsymbol{Y}}^{[\times]}\right)_{w}}$.

The inner product is defined as follows:

$\left(\hat{\boldsymbol{Y}}^{[a]}, \hat{\boldsymbol{Y}}^{[b]}\right)_{w}=\sum_{k=1}^{N} w_{k} \hat{\boldsymbol{y}}_{k}^{[a]} \hat{\boldsymbol{y}}_{k}^{[b]}, \quad(a, b=1, \ldots, d)$,

where $w_{k}=\min \{k, L, N-k-1\}$. Then based on these results it allows to perform a classification according to their disjoint relationship:

$\boldsymbol{Y} \approx \tilde{\boldsymbol{Y}}_{I_{1}}+\ldots+\tilde{\boldsymbol{Y}}_{I_{m}}$,

where $I_{l}(l=1, \ldots, m(\leq d))$ is defined as an index after grouping the reconstructed time series.

In change-point detection, by using the SSA, the reconstruction process is of importance since a structural difference between the subspaces in the original time series is qualitatively evaluated by the reconstructed time series. In the next subsection the definition of the SST will be explained.

\subsection{Singular spectrum transformation (SST)}

When trying to detect change points, typically, a parametric method such as the autoregressive model has often been applied (Di Bello et al., 1996). This kind of approach may not lead to a good result when the data series are heterogeneous and nonstationary because it is not sure whether it is appropriate to assume a certain stochastic model to such data. Thus it is reasonable to use a nonparametric method rather than a parametric one, as the first step of the data analysis. We suggest to use the SST for a change-point detection as it allows the nonparametric detection. 
Let us first put the reference time $t$ in a time series $\boldsymbol{Y}=$ $\left(y_{1}, \ldots, y_{N}\right)$ in order to divide it into two subtime series, $\boldsymbol{Y}^{(\mathrm{p})}$ of a "past part" and $\boldsymbol{Y}^{(\mathrm{f})}$ of a "future part" which can be defined as follows:

$\boldsymbol{Y}^{(\mathrm{p})}=\left(y_{t-b}, \ldots, y_{t-1}\right), \boldsymbol{Y}^{(\mathrm{f})}=\left(y_{t+\gamma}, \ldots, y_{t+\gamma+b-1}\right)$,

where $\gamma$ is a start point after the reference time $t$ for the future part and $b$ is defined as a size of both subtime series $(b<t)$, which are additional parameters besides the window length $L$ in the SSA. Since we assess the difference between both subtime series with the same length sitting next to each other in this study let $\gamma=0$. Then each trajectory matrix can be expressed as

$\mathbf{X}^{(\mathrm{p})}=\left[\boldsymbol{X}_{t-M}: \ldots: \boldsymbol{X}_{t-1-L}\right], \mathbf{X}^{(\mathrm{f})}=\left[\boldsymbol{X}_{\mathrm{t}}: \ldots: \boldsymbol{X}_{t+M-1}\right]$,

where $\boldsymbol{X}_{k}=\left\{y_{k}, \ldots, y_{k+L-1}\right\}(1 \leq k \leq M), L$ is defined as a window length for both time series, which means the number of elements in each vector, and then $M=b-L+1$. As expressed in Eqs. (2) and (3), both trajectory matrices are mathematically decomposed by SVD:

$$
\begin{aligned}
& \mathbf{X}^{(\mathrm{p})}=\mathbf{U}^{(\mathrm{p})} \mathbf{S}^{(\mathrm{p})} \mathbf{V}^{(\mathrm{p}) T}=\sum_{j=1}^{d} \sqrt{\lambda_{j}^{(\mathrm{p})}} \boldsymbol{U}_{j}^{(\mathrm{p})} \boldsymbol{V}_{j}^{(\mathrm{p}) T}, \\
& \mathbf{X}^{(\mathrm{f})}=\mathbf{U}^{(\mathrm{f})} \mathbf{S}^{(\mathrm{f})} \mathbf{V}^{(\mathrm{f}) T}=\sum_{j=1}^{d} \sqrt{\lambda_{j}^{(\mathrm{f})}} \boldsymbol{U}_{j}^{(\mathrm{f})} \boldsymbol{V}_{j}^{(\mathrm{f}) T} .
\end{aligned}
$$

According to the proposal by Idé and Inoue (2004, 2005), the structural difference between $\mathbf{X}^{(\mathrm{p})}$ and $\mathbf{X}^{(\mathrm{f})}$ can be sufficiently discussed by using the left singular vector, $\mathbf{U}^{(\mathrm{p})}=$ $\left[\boldsymbol{U}_{1}^{(\mathrm{p})}: \ldots: \boldsymbol{U}_{L}^{(\mathrm{p})}\right]$ and $\mathbf{U}^{(\mathrm{f})}=\left[\boldsymbol{U}_{1}^{(\mathrm{f})}: \ldots: \boldsymbol{U}_{L}^{(\mathrm{f})}\right]$ since the left singular vector $\boldsymbol{U}_{i}$ of the eigentriple explains the representative pattern in the trajectory matrix (Idé and Inoue, 2004, 2005). Hence by these left singular vectors, a hyperplane $\mathbf{U}_{l}$ and an eigenvector $\boldsymbol{\beta}$ are defined as follows:

$\mathbf{U}_{l}=\operatorname{span}\left\{\boldsymbol{U}_{1}^{(\mathrm{p})}, \ldots, \boldsymbol{U}_{l}^{(\mathrm{p})}\right\}, \quad \boldsymbol{\beta}=\boldsymbol{U}_{1}^{(\mathrm{f})}$,

where $l$ is the number of spanning vectors. To estimate the difference between the past and the future parts, $\boldsymbol{\beta}$ will be projected onto $\mathbf{U}_{l}$. Then a change-point (CP-) score is defined as follows:

$z=1-\sum_{i=1}^{l} \kappa(i, \beta)^{2}, \quad \kappa(i, \beta):=\beta^{T} U_{i}^{(\mathrm{p})}$,

where $0 \leq z \leq 1$ and $\kappa$ is the inner product of Eq. (11).

\subsection{Extended SST}

In the above conventional SST defined by Eqs. (11) and (12), the structural comparison is performed only for the most dominant representative pattern, $\boldsymbol{\beta}$ (mostly a trend term) of the future part on an arbitrary dimensional hyperplane of the past part, $\mathbf{U}_{l}$. Although the result is actually enough to understand the global behaviour of the change point in the data, the change point in the harmonics component which is extracted as another important property by the SSA, has not been considered so far. If the CP-score is calculated for further components it can be expected to find out a transitional time of the periodic term in the data. For that purpose we provide a new definition of the change-point detection technique based on the conventional SST and the multivariate SST (Tokunaga et al., 2011).

At the beginning, the whole time series is decomposed by the SSA into the reconstructed time series. Therefore, note that two different window length parameters will be introduced in this approach. One is used for the whole time series and the other for the reconstructed time series, which we call $L_{\mathrm{SSA}}$ and $L_{\mathrm{SST}}$ respectively. Then these reconstructed time series have to be grouped according to the results from singular value spectra and $w$ correlation as the representative patterns such as a trend, harmonics and noise, which are required to extend Eq. (11) as follows:

$\tilde{\mathbf{U}}_{I_{l}, l}=\operatorname{span}\left\{\hat{\boldsymbol{U}}_{I_{l}, 1}^{(\mathrm{p})}, \ldots, \hat{\boldsymbol{U}}_{I_{l}, l}^{(\mathrm{p})}\right\}, \quad \tilde{\boldsymbol{\beta}}_{I_{l}}=\hat{\boldsymbol{U}}_{I_{l}, 1}^{(\mathrm{f})}$,

where $I_{l}$ is a grouping index $(l=1, \ldots, m(\leq d)), m$ means the number of the groups, $l$ is the number of spanning vectors, and $\hat{\boldsymbol{U}}$ s mean the left singular vectors derived from the representative time series. Then the newly defined CP-score can be described by

$\tilde{z}_{I_{l}}=1-\sum_{i=1}^{l} \tilde{\boldsymbol{\beta}}_{I_{l}}^{T} \hat{\boldsymbol{U}}_{I_{l}, i}^{(\mathrm{p})}$

Grouping the reconstructed time series into representative time series, it might be expected that the change points of not only the trend component but also the harmonics' components included in the data can be detected.

\subsection{Statistical test}

As a statistical test for the results, we apply a Monte Carlo simulation by random shuffle (RS) surrogate, phase randomised (PR) surrogate, and iterative amplitude adjusted Fourier transform (iAAFT) surrogate methods (Scheinkman and LeBaron, 1989; Theiler et al., 1992; Schreiber and Schmitz , 2000).

The null hypothesises of these surrogates methods are typically defined as follows: (1) the observed time series is a sequence of independent and identically distributed (i.i.d.) random variables (for the RS surrogates), (2) the time series is stochastic data with a linear correlation (for the PR surrogates), and (3) the time series is generated from a nonlinear stochastic process. However, it is provided by performing a static monotonic nonlinear transformation (for the iAAFT surrogates). Table 1 shows whether the statistics, such as mean, variance, empirical distribution, and autocorrelation, of the original time series are preserved in the time series generated by these surrogate methods. 
Table 1. Comparison of the statistics of the original data with that of the surrogate data. $\mu:$ mean, $\sigma^{2}$ : variance. $\bigcirc$ : preserved, $\times:$ not preserved.

\begin{tabular}{lcccc}
\hline Surrogates & $\mu$ & $\sigma^{2}$ & Empirical dist. & Autocorrelation \\
\hline RS & $\bigcirc$ & $\bigcirc$ & $\bigcirc$ & $\times$ \\
PR & $\bigcirc$ & $\bigcirc$ & $\times$ & $\bigcirc$ \\
iAAFT & $\bigcirc$ & $\bigcirc$ & $\bigcirc$ & $\bigcirc$ \\
\hline
\end{tabular}

The significance test is performed by using the Monte Carlo technique. Since this method does not necessitate assuming any statistical distribution, it is appropriate to apply it to the result obtained by the nonparametric method such as the SSA. First, we generate a sufficiently large number of surrogate data. Next, we estimate the CP-scores of these surrogate data. Then we calculate each upper $95 \%$ quantile in the empirical distributions of the CP-scores as a threshold to validate the change point in this study. If the obtained $\mathrm{CP}$-score is higher than the threshold, we consider that each null hypothesis is rejected and the result is statistically significant.

\subsection{Model analysis}

In the following model we will assume that there exist changes in trends and harmonics extracted by means of SSA decomposition as the representative components. However, this method might also be useful to analyse a component with other kinds of dynamics like nonlinear changes. Although the remaining part is considered to be "noise", we suppose that it might contain the deterministic dynamics of the above representative components hidden in the noise part since the parameter choice of the number of the components is somehow arbitrary.

We consider the following synthetic example with two types of components which consist of linear and sine functions added by Gaussian noise $\epsilon_{t}$.

$\boldsymbol{Y}(t)=\alpha t+A \sin \left(\frac{2 \pi}{T} t\right)+\epsilon_{t}, \quad \epsilon \sim \mathcal{N}(0,1)$,

where $1 \leq t \leq 1000$,

$\alpha=\left\{\begin{array}{c}0.25(1 \leq t \leq 200) \\ -0.15(200<t \leq 550) \\ 0.35(550<t \leq 1000)\end{array}\right.$,

$T=\left\{\begin{array}{l}12(1 \leq t \leq 700) \\ 25(700<t \leq 1000)\end{array}\right.$

and $A=10$, which is illustrated in Fig. 5.

The synthetic data series contains change points in both linear and sine functions expressed in the first and the second terms on the right hand side in Eq. (15), which are at $t=200$ and $t=550$ for the linear functions and at $t=700$ for the sine functions.

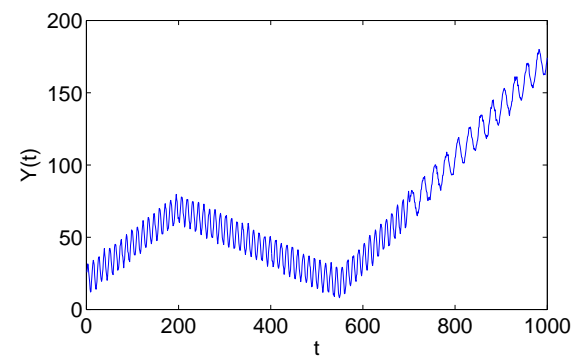

Fig. 5. Time series composed of the linear functions and the sine functions.
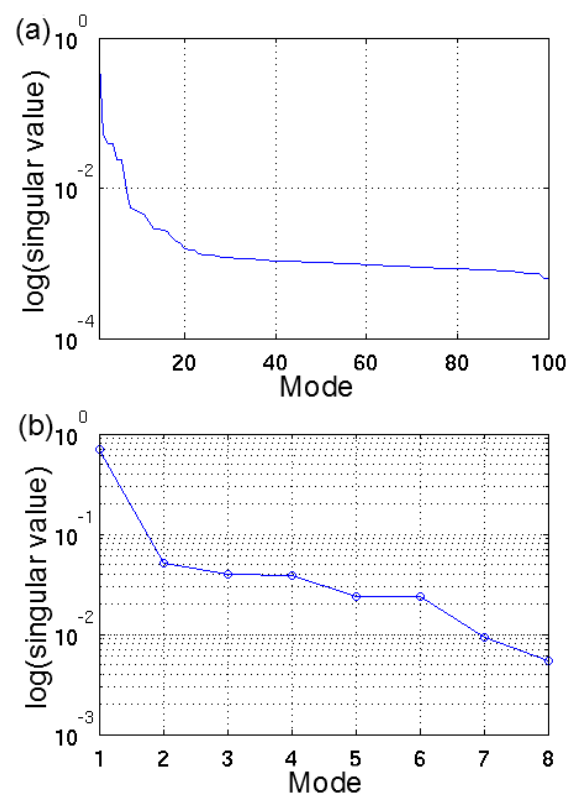

Fig. 6. Logarithmic singular value spectra expressed on percentage (a) for $L_{\mathrm{SSA}}=100$ and (b) for the first 8 modes.

In the first step, let us orthogonally decompose the time series. The window length parameter $L_{\mathrm{SSA}}$ is reasonably chosen between $\lfloor N / 10\rfloor$ and $\lfloor N / 2\rfloor$ ( $N$ is the length of time series) if there is not much information about the data. Thus, in this example, let $L_{\mathrm{SSA}}$ be $100(=\lfloor N / 10\rfloor)$.

The second step is to sort the decomposed time series into groups of representative time series. Basically, by the SSA decomposition, the first group corresponds to the trend component. The next groups are often categorised as harmonics term. And then the remaining components will be assumed as noise. According to the right hand side of Eq. (15), it is obvious that such groups correspond to their first term, second term, and third term, respectively. The determination of these groups from the results of the decomposition may be achieved by the singular value spectra and the $w$ correlation, Eq. (5). The first and the second mode differ in almost one magnitude (Fig. 6), followed by two pairs (3rd and 4th, and 5th and 6th), representing components with some periodicity. The 1 st mode contributes $78 \%$, the 2 nd mode $4.5 \%$, followed by rapidly decreasing contributions. Figure 7 
illustrates the pattern of $w$ correlation to discuss a separability of components. We separate the pattern into two parts consisting of the first 8 components and a residual part (9th and 100th components), where the first part is assumed as an interpretable part including trends and harmonics and the residual one is assumed as noise. Reconstructed time series of these 8 modes of the interpretable part are shown in Fig. 8. Figure $8 \mathrm{a}, \mathrm{b}$, and $\mathrm{g}$ show the local maximum more or less at $t=200$ and the local minimum more or less at $t=550$. Figure $8 \mathrm{c}$ and $\mathrm{d}$ show similar behaviours, which are drastically fluctuated until ca. $t=700$. In contrast, Fig. 8e and f show oscillation from $t=700$. In Fig. 8h relatively large fluctuations occur around $t=700$. Table 2 shows 6 groups of the first 3 strong correlated modes estimated by the $w$ correlation between the 1st and the 8th eigentriples. According to the table, since there are overlapping modes in these groups it allows to rearrange $\hat{I}_{i}(i=1, \cdots, 6)$ into the following new indices: $I_{1}:=\hat{I}_{1}=\hat{I}_{2}=\{1,2,7\}, I_{2}:=\hat{I}_{3}=\hat{I}_{4}=\{3,4,8\}$, and $I_{3}:=\hat{I}_{5}=\hat{I}_{6}=\{5,6,8\}$. This grouping implies to well extract the properties of the linear functions and the sine functions from the original time series defined in Eq. (15).

If it is assumed that the original time series consists of the trend, harmonics, and noise, it allows us to describe it by the following form:

$\boldsymbol{Y}=\tilde{\boldsymbol{Y}}_{I_{1}}+\tilde{\boldsymbol{Y}}_{\left(I_{2} \cup I_{3}\right)}+\tilde{\epsilon}$,

where

$\tilde{\boldsymbol{Y}}_{I_{1}}=\hat{\boldsymbol{Y}}_{1}+\hat{\boldsymbol{Y}}_{2}+\hat{\boldsymbol{Y}}_{7}$

$\tilde{\boldsymbol{Y}}_{\left(I_{2} \cup I_{3}\right)}=\hat{\boldsymbol{Y}}_{3}+\hat{\boldsymbol{Y}}_{4}+\hat{\boldsymbol{Y}}_{5}+\hat{\boldsymbol{Y}}_{6}+\hat{\boldsymbol{Y}}_{8}$,

and $\tilde{\epsilon}$ is the residual. Let us here define $\tilde{\boldsymbol{Y}}_{I_{1}}$ and $\tilde{\boldsymbol{Y}}_{\left(I_{2} \cup I_{3}\right)}$ as representative time series. Note that $\tilde{\boldsymbol{Y}}_{\left(I_{2} \cup I_{3}\right)}$ is, however, reconstructed by using the unique values from both $I_{2}$ and $I_{3}$ in order to avoid duplication of the component. Figure 9 illustrates the linear functions and the sine functions defined in Eq. (15) in (a) and (b) and the representative time series $\tilde{\boldsymbol{Y}}_{I_{1}}$ and $\tilde{\boldsymbol{Y}}_{\left(I_{2} \cup I_{3}\right)}$ in (c) and (d). These results show that $\tilde{\boldsymbol{Y}}_{I_{1}}$ and $\tilde{\boldsymbol{Y}}_{\left(I_{2} \cup I_{3}\right)}$ well reconstruct both functions, which are defined as the trend and the harmonics terms, respectively.

In the third step the CP-score will be computed by the SST. For the SST technique there are the following four parameters: (i) a time length of both past and future parts $b$, (ii) a start position of the subtime series in the future part $\gamma$ from the reference time $t$, (iii) a window length for these subtime series $L_{\mathrm{SST}}\left(\leq b<L_{\mathrm{SSA}}\right)$, and (iv) the number of the left singular vectors of the past part to span a hyperplane $l$. Although a proper rule of the parameter setting is not yet regulated, as several reasonable values we choose $b_{1}=50, b_{2}=60$, $b_{3}=70, b_{4}=80, b_{5}=90$, and $b_{6}=100$ in this study. The $\gamma$ is fixed by zero since the structural difference of the neighbourhood will be focused on in this study. The $L_{\text {SST }}$ must be determined by a value less than or equal to $\lfloor b / 2\rfloor$. Here let us choose that $L_{\mathrm{SST}}$ is a half of each $b$. Then consider the hyperplane for the $l=2,3$ (Table 3).
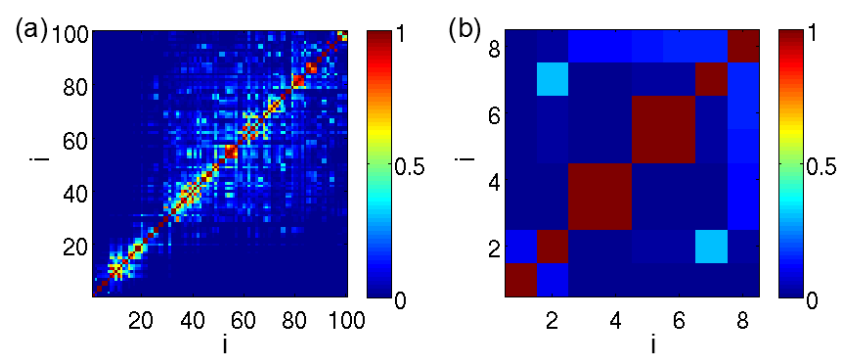

Fig. 7. $w$ correlation, (a) for the whole components for $L_{\mathrm{SSA}}=100$ and (b) for the first 8 components.

Table 2. Relationship of modes between the 1 st and 8 th eigentriples by $w$ correlation. $\hat{I}_{i}(i=1, \cdots, 6)$ : group indices.

\begin{tabular}{ccccccc}
\hline & $\hat{I}_{1}$ & $\hat{I}_{2}$ & $\hat{I}_{3}$ & $\hat{I}_{4}$ & $\hat{I}_{5}$ & $\hat{I}_{6}$ \\
\hline \multirow{4}{*}{$\operatorname{mode}$} & 1 & 2 & 3 & 4 & 5 & 6 \\
& 2 & 7 & 4 & 3 & 6 & 5 \\
& 7 & 1 & 8 & 8 & 8 & 8 \\
\hline
\end{tabular}

Table 3. Parameter combinations of the example for SST. $b$ : a time length of both past and future parts, $\gamma$ : a start position of the subtime series in the future part from the reference time, $L_{\mathrm{SST}}$ : a window length for these subtime series, $l$ : the number of the left singular vectors of the past part to span a hyperplane.

\begin{tabular}{ccccc}
\hline & $b$ & $\gamma$ & $L_{\text {SST }}$ & $l$ \\
\hline (a) & 50 & 0 & 25 & $\{2,3\}$ \\
(b) & 60 & 0 & 30 & $\{2,3\}$ \\
(c) & 70 & 0 & 35 & $\{2,3\}$ \\
(d) & 80 & 0 & 40 & $\{2,3\}$ \\
(e) & 90 & 0 & 45 & $\{2,3\}$ \\
(f) & 100 & 0 & 50 & $\{2,3\}$ \\
\hline
\end{tabular}

Figures 10 and 11 show the results of the $\mathrm{CP}$-scores for the trend and harmonics terms. The panels (a)-(f) in both figures explain the results for each parameter combination in table 3 .

From these results we get that $\tilde{\boldsymbol{Y}}_{I_{1}}$ and $\tilde{\boldsymbol{Y}}_{\left(I_{2} \cup I_{3}\right)}$ are corresponding to the trend term and the harmonics term, respectively since the significant peaks in Fig. 10 are shown at time 200 and 500, and the significant peak in Fig. 11 is shown at time 700 as defined in Eqs. (15) and (16).

In the Monte Carlo significance test, we test the result for the parameters, $b=60, L_{\mathrm{SST}}=30$, and $l=3$. Figures 12 and 13 show the CP-scores and the thresholds estimated by the above introduced surrogate data methods in (a), (b), and (c) and then detecting the statistically significant change points by the red bars in (d), (e), and (f). As a result, the significant peaks of the CP-score for $\tilde{\boldsymbol{Y}}_{I_{1}}$ and $\tilde{\boldsymbol{Y}}_{\left(I_{2} \cup I_{3}\right)}$ can be detected at around 200 and 550 (the linear functions) and at around 700 (the sine functions) with $95 \%$ confidence, which coincide very well with the points set up in Eq. (15). 

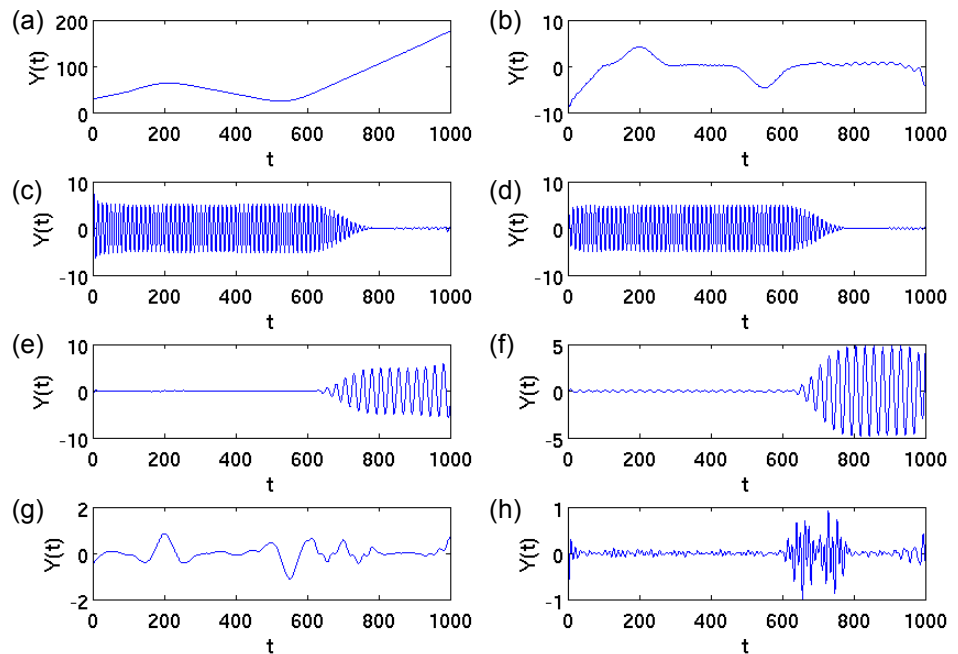

Fig. 8. Reconstructed time series. (a) for 1 st mode, (b) for 2 nd mode, (c) 3rd mode, (d) 4th mode, (e) 5 th mode, (f) 6 th mode, (g) 7 th mode, and (h) 8th mode.
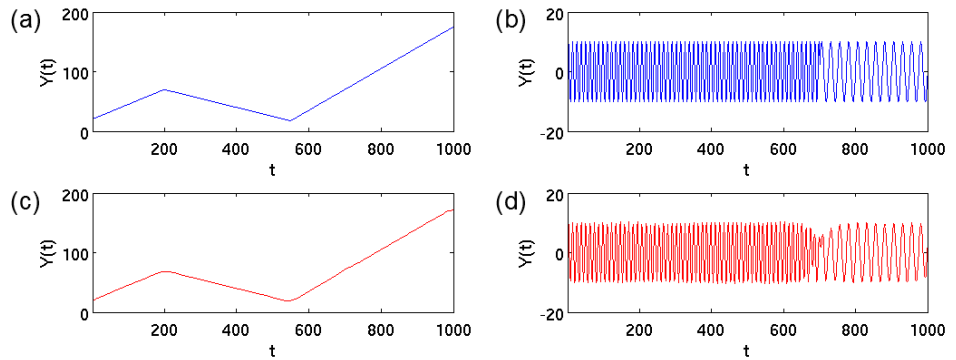

Fig. 9. The realisations of (a) $\alpha t$ and (b) $A \sin \left(\frac{2 \pi}{T} t\right)$ in Eq. (15), and the representative time series of (c) $\tilde{\boldsymbol{Y}}_{I_{1}}$ and (d) $\tilde{\boldsymbol{Y}}_{I_{2}}$.
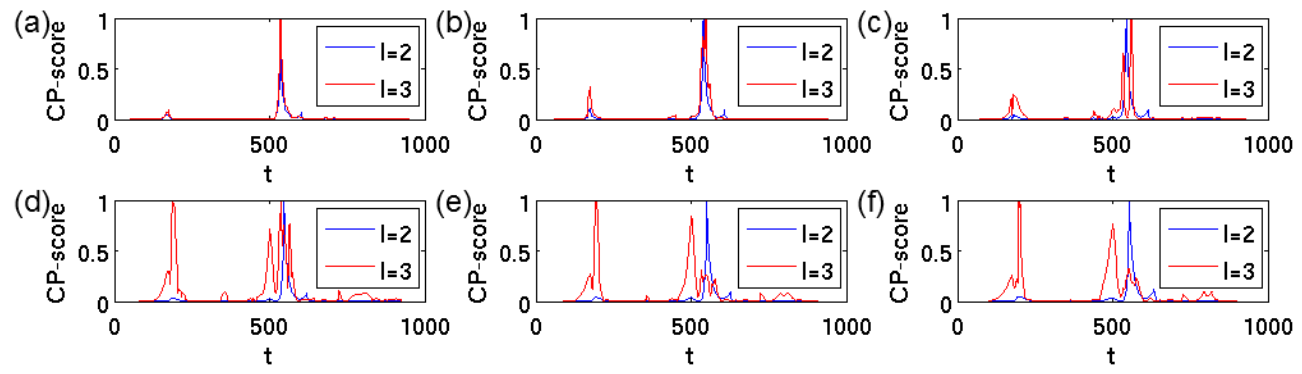

Fig. 10. CP-scores of $\tilde{\boldsymbol{Y}}_{1}$. (a) $b=50, L_{\mathrm{SST}}=25$, (b) $b=60, L_{\mathrm{SST}}=30$, (c) $b=70, L_{\mathrm{SST}}=35$, (d) $b=80, L_{\mathrm{SST}}=40,(\mathbf{e}) b=90, L_{\mathrm{SST}}=$ 45 , and (f) $b=100, L_{\mathrm{SST}}=50$, and then always $\gamma=0$ and $l=2$ (blue line), 3 (red line).
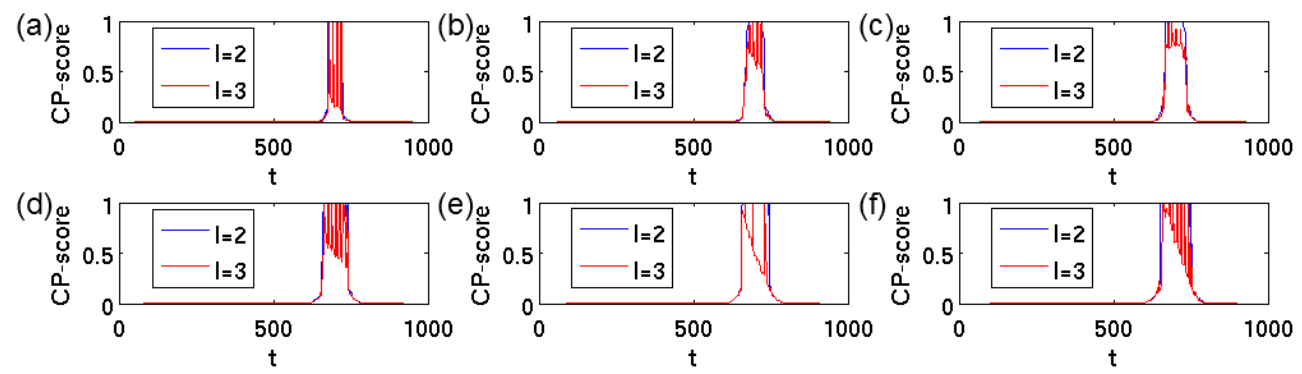

Fig. 11. CP-scores of $\tilde{\mathbf{Y}}_{2}$. (a) $b=50, L_{\mathrm{SST}}=25$, (b) $b=60, L_{\mathrm{SST}}=30$, (c) $b=70, L_{\mathrm{SST}}=35$, (d) $b=80, L_{\mathrm{SST}}=40$, (e) $b=90, L_{\mathrm{SST}}=$ 45 , and (f) $b=100, L_{\mathrm{SST}}=50$, and then always $\gamma=0$ and $l=2$ (blue line), 3 (red line). 


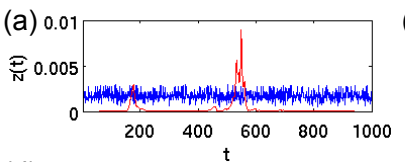

(d)

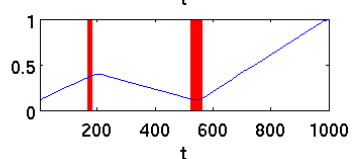

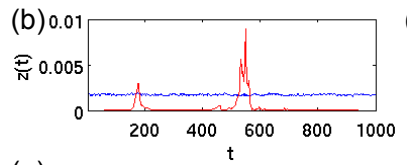

(e)

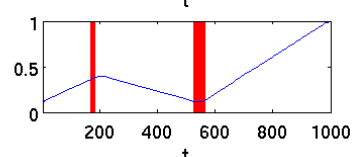

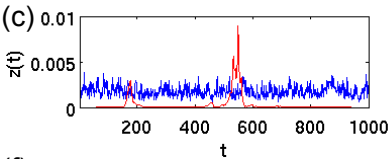

(f)

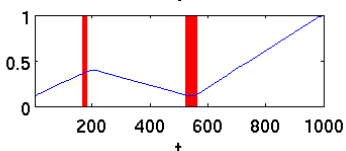

Fig. 12. (a), (b), and (c) are the CP-scores of the trend term (the red line) and the $95 \%$ confidence intervals estimated in the Monte Carlo significance test by the (a) RS, (b) PR, and (c) iAAFT, respectively (the blue line). (d), (e), and (f) are the time series of the trend term (the blue line) and the change points which have the statistic significance (the red bars).

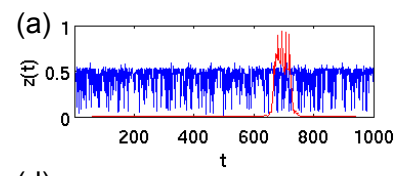

(d)

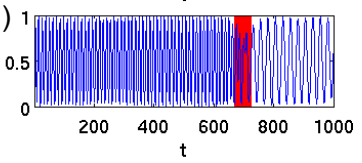

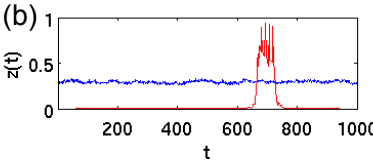

(e)

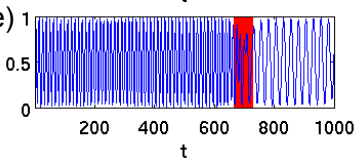

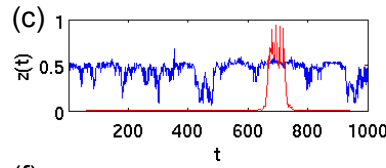

(f)

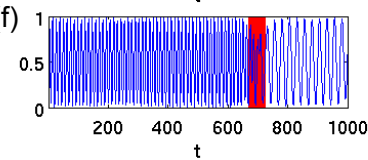

Fig. 13. (a), (b), and (c) are the CP-scores of the harmonics term (the red line) and the $95 \%$ confidence intervals estimated in the Monte Carlo significance test by the (a) RS, (b) PR, and (c) iAAFT, respectively (the blue line). (d), (e), and (f) are the time series of the harmonics term (the blue line) and the change points which have the statistic significance (the red bars).
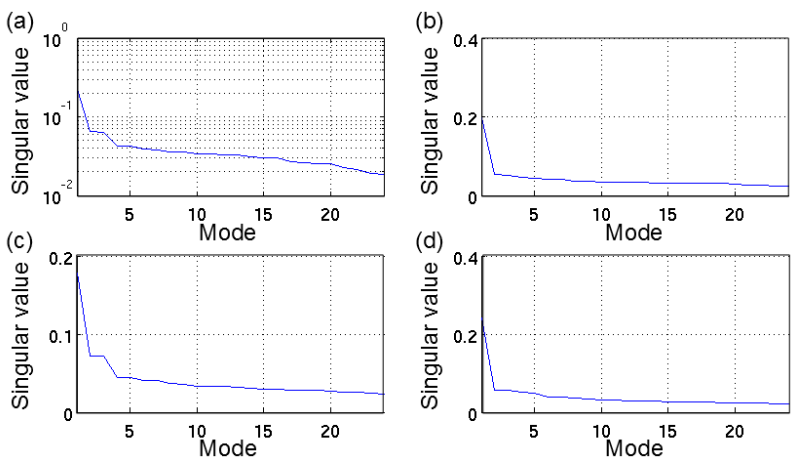

Fig. 14. Singular value spectra (in percentage) with the 24 modes. (a) Nakuru, (b) Naivasha, (c) Narok, and (d) Kisumu. The 1st mode of each result shows the highest rate which means the most dominant mode.
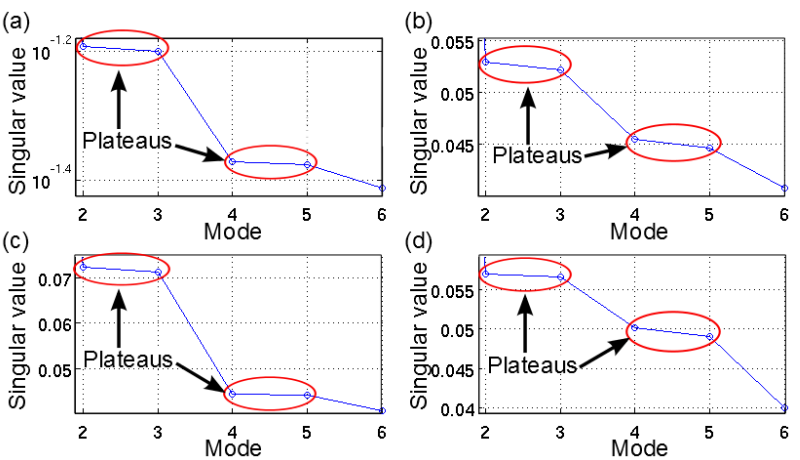

Fig. 15. Singular value spectra (in percentage) between the 2 nd mode and the 6th mode magnified from Fig. 14. (a) Nakuru, (b) Naivasha, (c) Narok, and (d) Kisumu. In all the stations, there are two pairs between the 2 nd and the 3rd modes and between the 4th and the 5th modes (red ellipses).

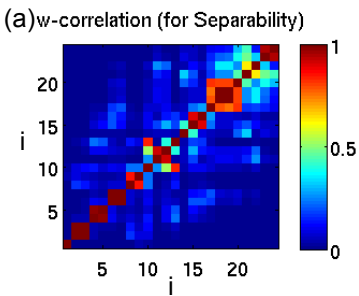

(c) w-correlation (for Separability)

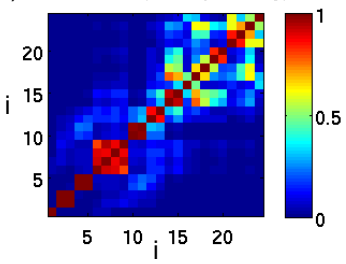

(b) w-correlation (for Separability)

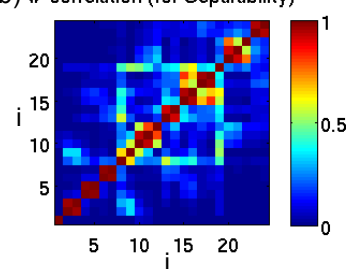

(d) w-correlation (for Separability)

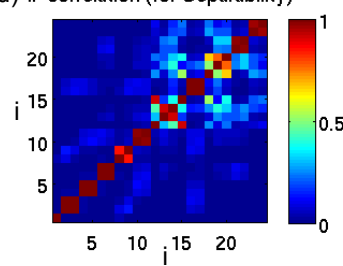

Fig. 16. $w$ correlation with the 24 reconstructed time series. (a) Nakuru, (b) Naivasha, (c) Narok, and (d) Kisumu. When the mode for the reconstructed time series is higher, the separability becomes weaker because more different colour cells indicating the correlation with other modes are illustrated in the row and column directions of each reconstructed time series in the results.

\section{Change-point detection of precipitation in East Africa}

This section will be devoted to the application of our method to the monthly Kenyan precipitation illustrated in Figs. 3 and 4 . First, the precipitation will be orthogonally decomposed by the SSA. We choose any number related to the annual cycle (12-month) for the window length parameter $L_{\mathrm{SSA}}$ since the original time series include a seasonality. Thus, in this study $L_{\mathrm{SSA}}=24$ (for $2 \mathrm{yr}$ ). When the grouping and 

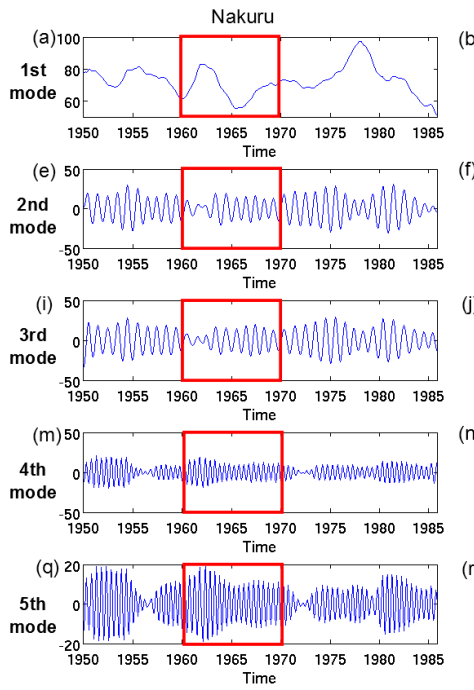
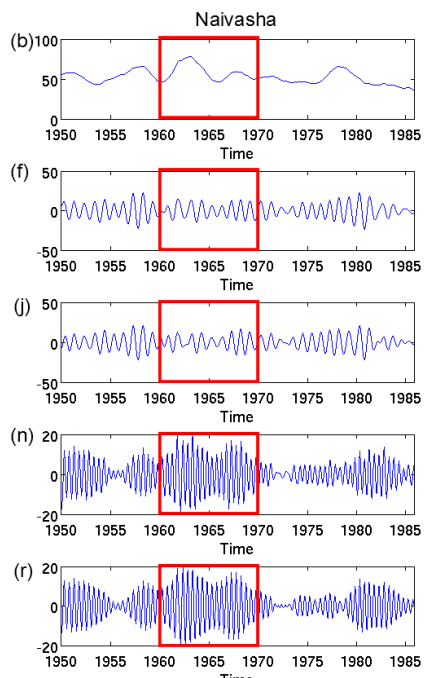
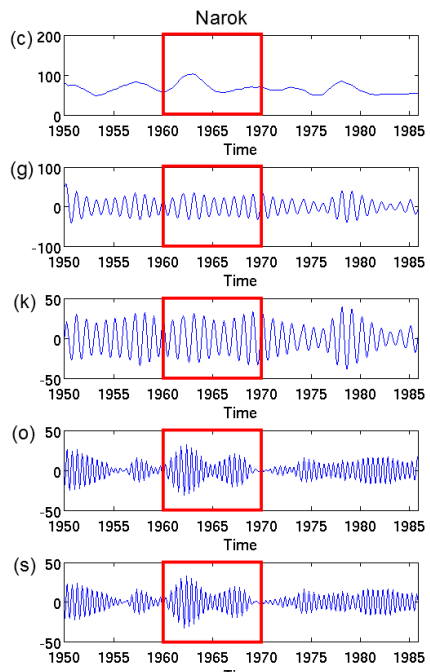
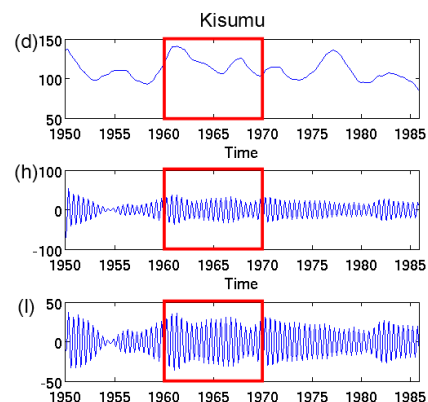

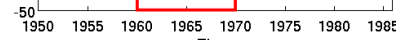
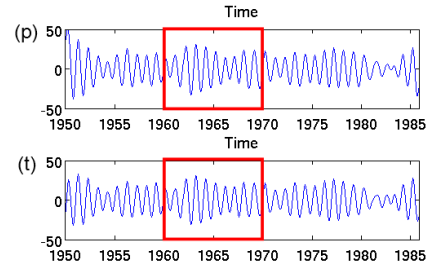

Fig. 17. First 5 reconstructed time series by SSA. (a, e, i, m, q) for Nakuru. (b, f, j, n, r) for Naivasha. (c, g, k, o, s) for Narok. (d, h, l, p, t) for Kisumu. (a-d) show the slowly varying trend components. (e-t) show the frequent components. The red rectangles between 1960 and 1969 mean the interesting decade in this study.
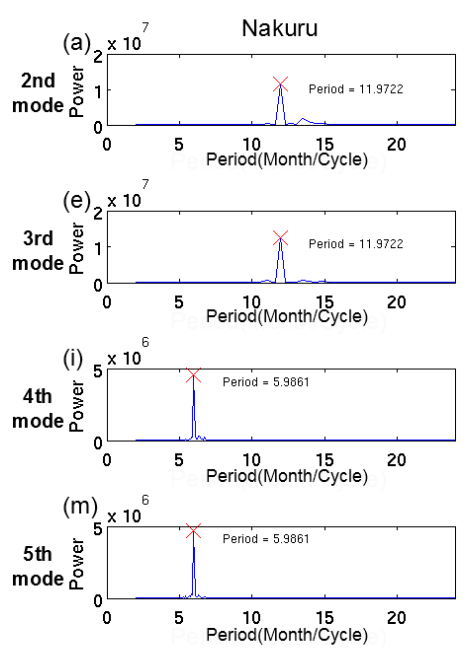
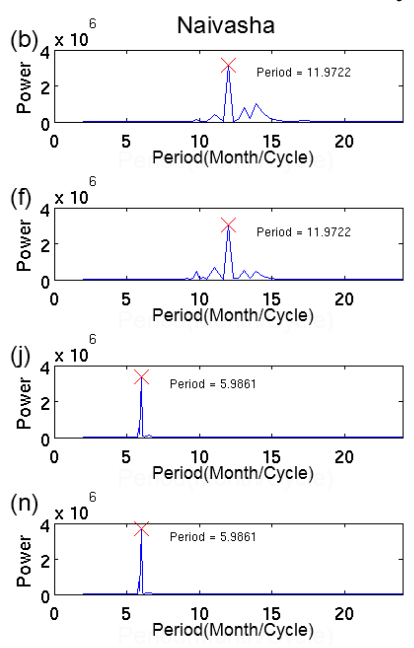
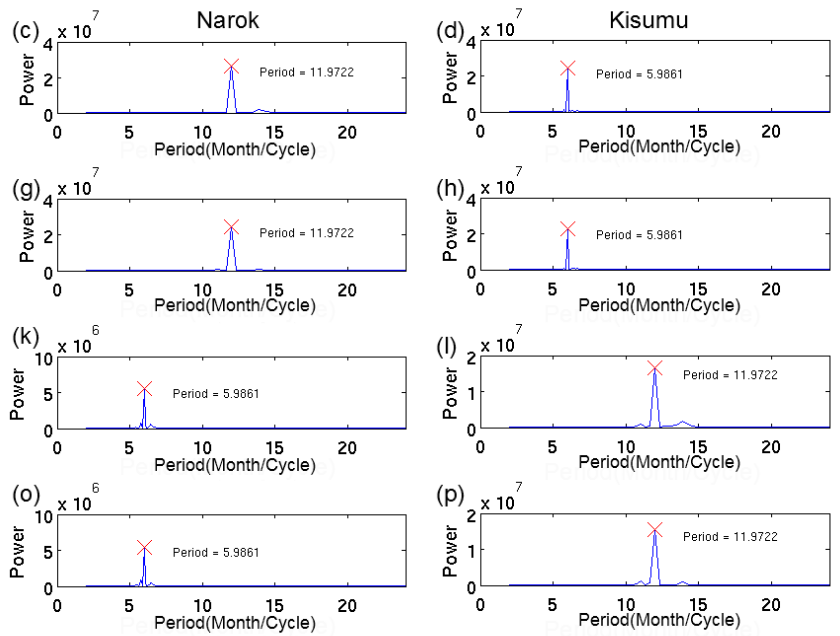

Fig. 18. Periodicities of the extracted harmonics components shown in (e-t) in Fig. 17. (a, e, i, m) for Nakuru. (b, f, j, ,n) for Naivasha. (c, $\mathbf{g}$, $\mathbf{k}$, and $\mathbf{o}$ ) for Narok. (d, h, l, and p) for Kisumu. The two different periodicities are estimated, which are about the annual cycle (12 months) and the semiannual cycle (6 months).

separability of the reconstructed time series are discussed by the parameter choice, the results of singular value spectra and $w$ correlation will provide reasonable information in the sense of climate cycles. For example, in Figs. 14 and 16, which show the results of the singular value spectra and the $w$ correlation for $L_{\mathrm{SSA}}=24$, the reconstructed time series from the 1 st to the 5 th modes can basically be characterised as representative time series. These reconstructed time series are illustrated in Fig. 17. Then from these results, let us consider the grouping of the reconstructed time series into the representative time series.
First, all the results of the singular value spectra in Fig. 14 show the relatively high values for the 1 st mode. From the results of $w$ correlations in Fig. 16, we consider that there is no mode showing the high correlation with the 1st mode since that of the closest mode to the 1st mode is at most less than 0.1 in all the stations. In addition, since the behaviours of the reconstructed time series of the 1st mode shown in Fig. $17 \mathrm{a}-\mathrm{d}$ are slowly varying, we assume it as the representative time series of the trend components. Second, according to the result in Fig. 15, modes appear pairwise, i.e. 2nd + $3 \mathrm{rd}$, and 4 th +5 th; thus indicating the harmonic behaviours 


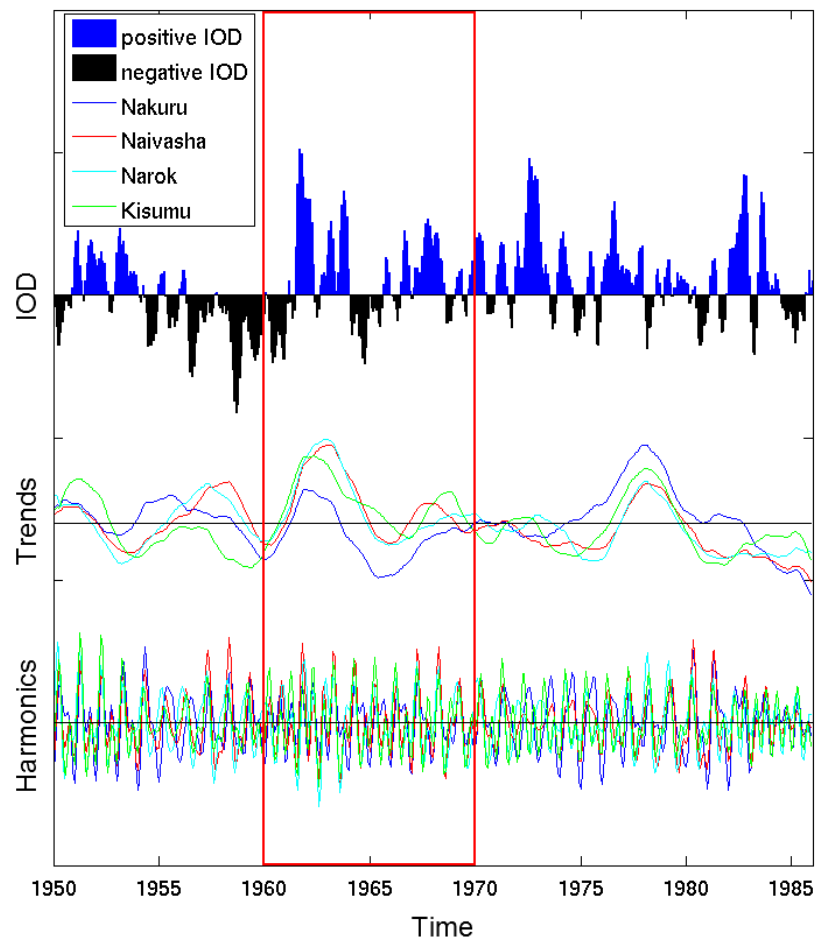

Fig. 19. IOD and the two representative time series (trend and harmonics) of the monthly precipitations from 1950 to 1985 . This study focuses on the events of IOD around the early 1960s showing the change from the negative mode to the positive mode. At that time, the precipitations in all the stations immediately increased according to the trend time series and the periodicity is changed from a 12-month cycle to 6-month cycle in Nakuru, Naivasha, and Kisumu according to the harmonics time series (see Table 5).

of these modes. In Fig. 16, $w$ correlations corresponding to these pairs are both more than 0.9. The behaviours of the reconstructed time series shown in Fig. 17e-t are modulated oscillations, which almost indicate the annual cycle and the semiannual cycle according to Fig. 18. Therefore, we assume the sum of them as a representative time series of harmonics components. To visually compare these two representative time series with the IOD, Fig. 19 shows them simultaneously.

Next, the extended SST will be applied to these representative time series. As with the parameter choice in the window length $L_{\mathrm{SSA}}$, the other parameters $b, \gamma, L_{\mathrm{SST}}$, and $l$ should be determined based on the seasonality. In this study $L_{\mathrm{SSA}}$ is fixed by 24 (for $2 \mathrm{yr}$ ), and the other parameter combinations are set as listed in Table 4.

We repeatedly apply the Monte Carlo significance test to the change-point scores 100 times. These results are shown in Figs. A1 and A2. Furthermore, to discuss properties of the harmonics term, Figs. 20-23 show the transition of these periodicities since the change point in the representative time series of the harmonics is assumed as a point where a periodicity changes. However, this study focuses on the
Table 4. Parameter combinations of the precipitations for SST. $b$ : a time length of both past and future parts, $\gamma$ : a start position of the subtime series in the future part from the reference time, $L_{\mathrm{SST}}$ : a window length for these subtime series, $l$ : the number of the left singular vectors of the past part to span a hyperplane.

\begin{tabular}{ccccc}
\hline & $b$ & $\gamma$ & $L_{\text {SST }}$ & $l$ \\
\hline$(1)$ & 12 & 0 & 6 & 2 \\
$(2)$ & 24 & 0 & 12 & 2 \\
$(3)$ & 36 & 0 & 18 & 2 \\
$(4)$ & 48 & 0 & 24 & 2 \\
$(5)$ & 60 & 0 & 30 & 2 \\
$(6)$ & 72 & 0 & 36 & 2 \\
\hline
\end{tabular}

Table 5. Monthly periodicities every five years of the representative harmonics time series [month] from 1950 to 1984 . The main periodicities in Nakuru, Naivasha, and Kisumu transiently change from annual cycle to semiannual cycle in the 1960s.

\begin{tabular}{ccccc}
\hline Time region & Nakuru & Naivasha & Narok & Kisumu \\
\hline $1950-1954$ & 11.9 & 11.9 & 11.9 & 5.9 \\
$1955-1959$ & 11.9 & 11.9 & 11.9 & 11.9 \\
$1960-1964$ & 5.9 & 5.9 & 11.9 & 5.9 \\
$1965-1969$ & 11.9 & 5.9 & 11.9 & 5.9 \\
$1970-1974$ & 11.9 & 11.9 & 11.9 & 5.9 \\
$1975-1979$ & 11.9 & 11.9 & 11.9 & 11.9 \\
$1980-1984$ & 11.9 & 5.9 & 5.9 & 5.9 \\
\hline
\end{tabular}

turn of events from the negative IOD to the positive IOD, thus we estimate the periodicities around 1960 in each station ((a) 1950-1954, (b) 1955-1959, (c) 1960-1964, and (d) 1965-1969).

\section{Discussion}

We demonstrate that our method successfully detects the change points in the representative time series extracted from the synthetic data series of Eq. (15) and from the actual precipitation in Kenya as a climate data analysis of East Africa. Since the relationship among these representative time series reconstructed by SSA is orthogonal we can state that the results of change-point detection are the independent and different properties in the data.

In the synthetic data series, the representative time series of trend corresponds to the linear functions, where the change points can be detected at $t=200$ and at $t=550$ as shown in Fig. 12. Similarly, that of harmonics corresponds to the sine functions, where the change point can be detected at $t=700$ as shown in Fig. 13. They approximately coincide with the definition of Eq. (16). 

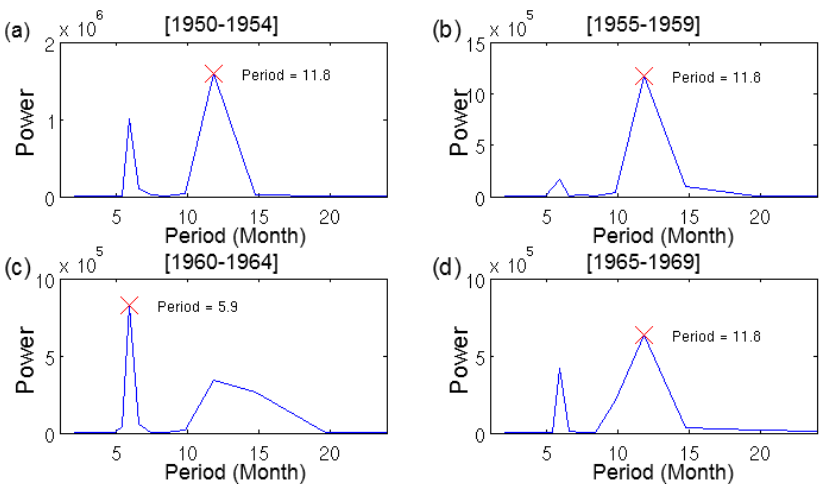

Fig. 20. Periodicities around 1960 in Nakuru estimated from the representative time series of the harmonics. (a) shows the periodicities between 1950 and 1954, (b) between 1955 and 1959, (c) between 1960 and 1964, and (d) between 1965 and 1969. The red cross mark means the major periodicity in each time region.
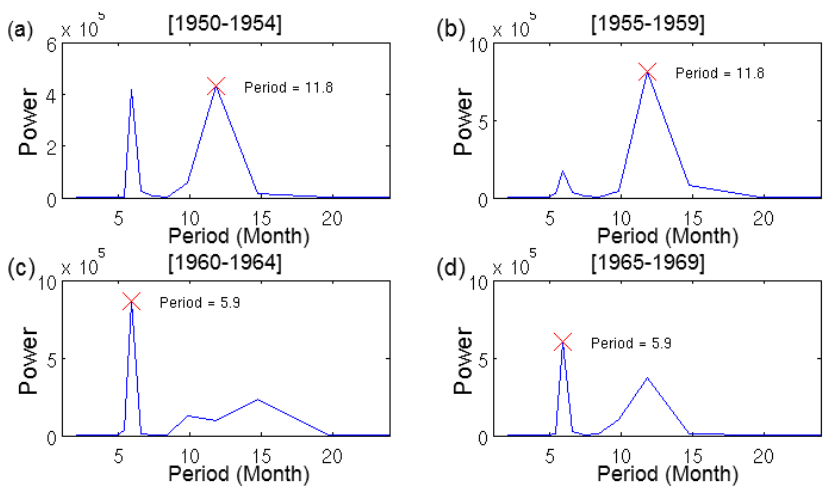

Fig. 21. Periodicities around 1960 in Naivasha estimated from the representative time series of the harmonics. (a) shows the periodicities between 1950 and 1954, (b) between 1955 and 1959, (c) between 1960 and 1964, and (d) between 1965 and 1969. The red cross mark means the major periodicity in each time region.

In the application to the actual precipitation in Kenya the results of the trend terms show the significant variations of all the data around 1960 (see Fig. A1). However the result for the parameter combination (6) $\left\{b=72, \gamma=0, L_{\mathrm{SST}}=\right.$ $36, l=2\}$ listed in Table 4 is different from the others. The reason might be that the parameter $b$ is too long to detect the change point at 1960 . With regards to the harmonics terms, the significant change points are often estimated also in 1960s (see Fig. A2). According to the periodograms of $10 \mathrm{yr}$ before and after 1960 shown in Figs. 20-23, the strongest power of periodicity changes from a 12-month cycle to 6-month cycle in Nakuru, Naivasha, and Kisumu. Although the strongest one in Narok does not change we can see, however, that the power of a 6-month cycle at least increases between 1955-1959 and 1960-1964.

We discuss the interpretations of the results in climatological context by linking them with IOD. We have found a
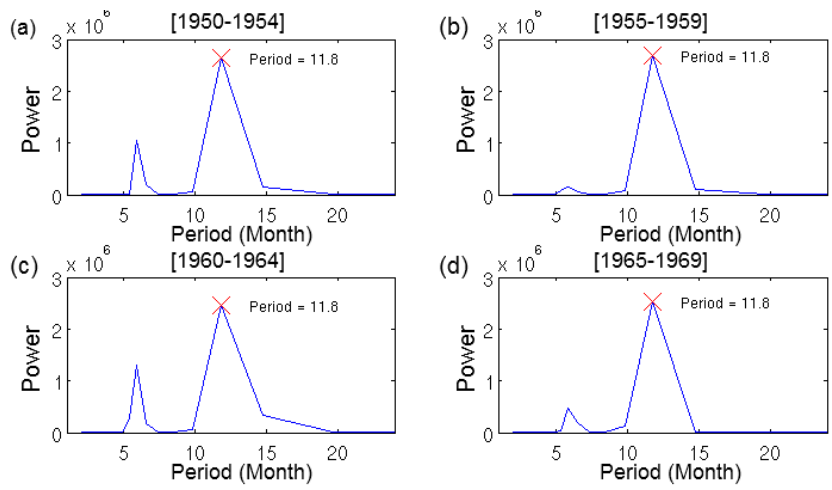

Fig. 22. Periodicities around 1960 in Narok estimated from the representative time series of the harmonics. (a) shows the periodicities between 1950 and 1954, (b) between 1955 and 1959, (c) between 1960 and 1964, and (d) between 1965 and 1969. The red cross mark means the major periodicity in each time region.
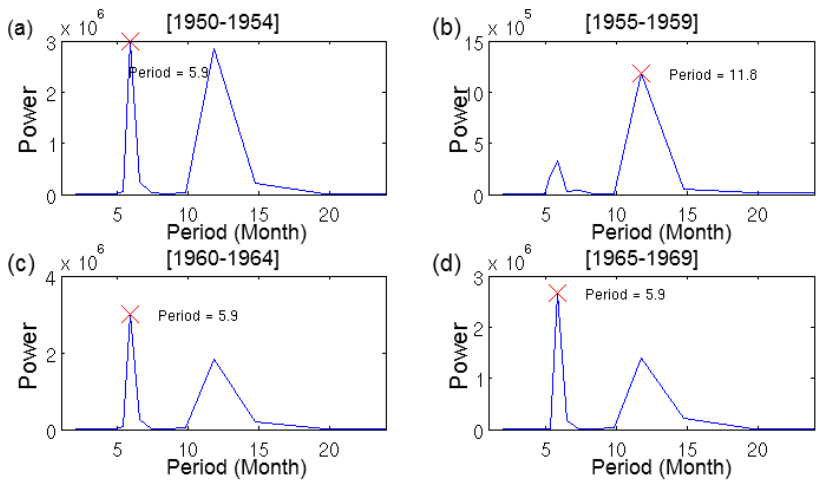

Fig. 23. Periodicities around 1960 in Kisumu estimated from the representative time series of the harmonics. (a) shows the periodicities between 1950 and 1954, (b) between 1955 and 1959, (c) between 1960 and 1964, and (d) between 1965 and 1969. The red cross mark means the major periodicity in each time region.

significant change point occurring in the 1960s. During this period, the phenomenon of IOD has drastically changed between the negative mode and positive mode. According to Fig. 19, especially, to the trend time series, we can see that the heavy rainfalls are provided in the early 1960s and afterwards the rainfalls are immediately decreasing for several years. This result coincides with the record-breaking floods in East Africa in 1961 (Behera et al., 2005). From the result of the harmonics time series, the cycle of rainfalls in Nakuru, Naivasha, and Kisumu decreased from 12-month to 6-month in the early 1960s (see Table 5). This corresponds to increased rainfalls in the usually short rainy season in autumn, which was then comparable to the long rainy season in spring in 1961. 

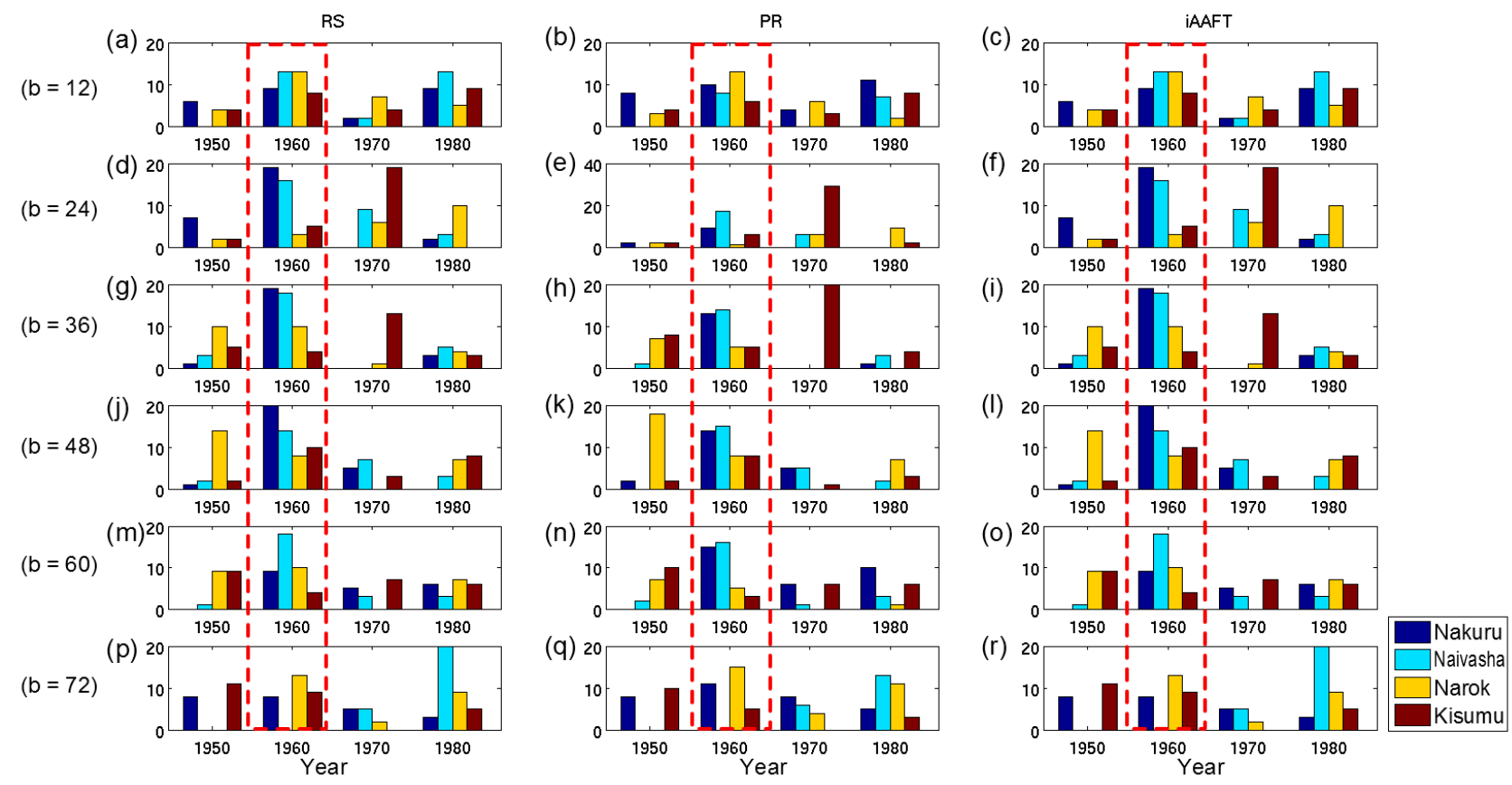

Fig. A1. Histogram, classified into the 1950s, 1960s, 1970s, and 1980s, of the significant change points of the trend estimated by using the three kinds of surrogate methods: RS (left panels), PR (middle panels), and iAAFT (right panels). (a-c) for $b=12$, (d-f) for $b=24,(\mathbf{g}-\mathbf{i})$ for $b=36$, (j-l) for $b=48,(\mathbf{m}-\mathbf{o})$ for $b=60$, and $(\mathbf{p}-\mathbf{r})$ for $b=72$. Blue bars mean Nakuru, light blue ones Naivasha, yellow ones Narok, and red ones Kisumu.
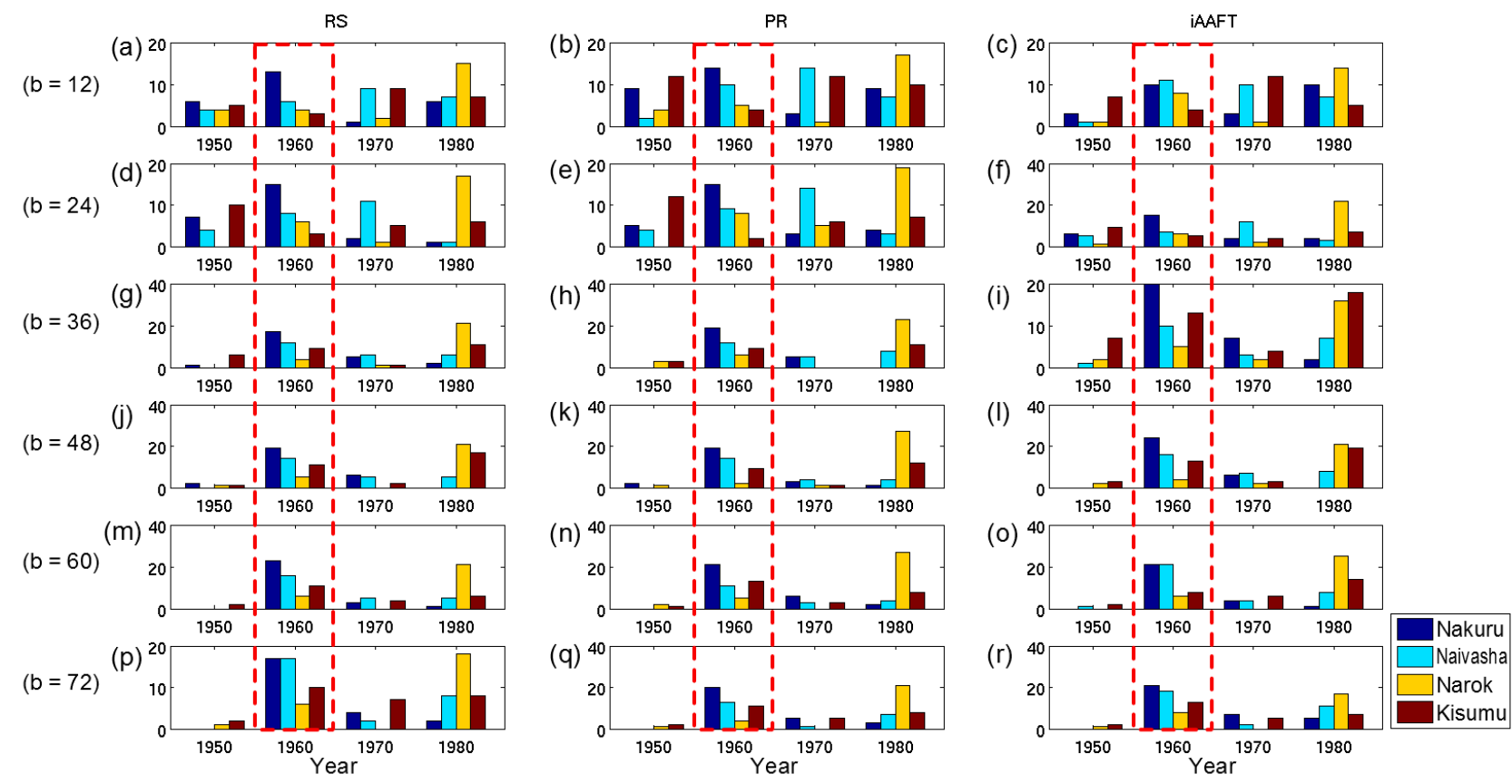

Fig. A2. Histogram, classified into the 1950s, 1960s, 1970s, and 1980s, of the significant change points of the harmonics estimated by using the three kinds of surrogate methods: RS (left panels), PR (middle panels), and iAAFT (right panels). (a-c) for $b=12$, (d-f) for $b=24$, $(\mathbf{g}-\mathbf{i})$ for $b=36,(\mathbf{j}-\mathbf{l})$ for $b=48,(\mathbf{m}-\mathbf{o})$ for $b=60$, and $(\mathbf{p}-\mathbf{r})$ for $b=72$. Blue bars mean Nakuru, light blue ones Naivasha, yellow ones Narok, and red ones Kisumu. 


\section{Conclusions}

In this paper we proposed to extend the SST-based method of change-point detection in order to consider nonlinear change points and applied it to the precipitations in Kenya. The aim of this study is to identify the change points in the representative time series of the trend as well as the harmonics extracted from the original time series. First, the change point detected in the time series of the trend suggested the occurrence of drastic change in slope from a negative direction to a positive one and vice versa. Second, from the result of change-point detection in the time series of the harmonics the point where the major period in the time series of the harmonics switched could be estimated.

In the application of our method to the precipitations in Kenya, we suggested the change points of all the data are detected in the early 1960s according to the results of their scores, where the behaviour of IOD is similar to the representative time series of the trend extracted by our method. From the harmonics time series we suggested that since the precipitation amount of the short rainy season in Kenya immediately increased by the positive IOD, the periodicity of rainfalls became shorter in the early 1960s and therefore it was detected as the change point. The next challenge in this work is to ensure more accurate understanding of the climate change in East Africa by applying our method to other data series related to this issue.

\section{Appendix A}

\section{Histograms of the significant change points}

In the following we present the results of the significance test for the detection of change points in the considered four decades. Figures A1 and A2 show the histograms of the detected significant change points for the trend term and the harmonics term, respectively, in the decades classified into the 1950s, 1960s, 1970s, and 1980s: (a-c) for the parameter combination as given in Table 4; (1), (d-f) for (2), $(\mathbf{g}-\mathbf{i})$ for (3), (j-l) for (4), (m-o) for (5), and (p-r) for (6), of the three surrogate methods (described by RS, PR, and iAAFT).

Acknowledgements. The data sets of IOD, NINO3 index, and Kenyan precipitation used in this study were provided by the Japan Agency for Marine Earth Science and Technology (http://www.jamstec.go.jp), KNMI Climate Explorer (http://climexp.knmi.nl), and GHCN v2 database (http://www.ncdc.noaa.gov/), respectively. The authors would like to thank Udo Schwarz (Center for Dynamics of Complex Systems, University of Potsdam) and Tsuyoshi Idé (IBM Tokyo Research Laboratory) for giving valuable comments and suggestions.

Edited by: J. Kurths

Reviewed by: two anonymous referees

\section{References}

Ashok, K., Guan, Z., and Yamagata, T.: Impact of the Indian Ocean Dipole on the relationship between the Indian Monsoon rainfall and ENSO, Geophys. Res. Lett, 26, 4499-4502, 2001.

Basseville, M. and Nikiforov, I.: Detection of abrupt changes, theory and application, Englewood Cliffs NJ, Prentice-Hall, 1993.

Behera, S. K., Luo, J. J., Masson, S., Delecluse, P., Gualdi, S., Navarra, A., and Yamagata, T.: Paramount impact of the Indian Ocean Dipole on the East African short rains : a CGCM study, J. Climate, 18, 4514-4530, 2005.

Bishop, C. M.: Pattern recognition and machine learning, Springer, 738 pp., 2007.

Björnsson, H. and Venegas, S. A.: A manual for EOF and SVD analyses of climate data, Centre for Climate and Global Change Research, Rep. 97, 53 pp., 1997.

Brockwell, P. J. and Davis, R. A.: Time series: theory and methods, Springer, 584 pp., 1991.

Brockwell, P. J. and Davis, R. A.: Introduction to time series and forecasting, Springer, 434 pp., 2002.

Di Bello, G., Lapenna, V., Macchiato, M., Satriano, C., Serio, C. and Tramutoli, V.: Parametric time series analysis of geoelectrical signals: an application to earthquake forecasting in Southern Italy, Ann. Geofis., 39, 11-21, 1996.

Elsner, J. B. and Tsonis, A. A.: Singular Spectrum Analysis, A New Tool in Time Series Analysis, Plenum Press, 164 pp., 1996.

Flohn, H.: Rainfall teleconnections in northern and northeastern Africa, Theor. Appl. Climatol., 38, 191-197, 1987.

Ghil, M., Allen, M. R., Dettinger, M. D., Ide, K., Kondrashov, D., Mann, M. E., Robertson, A. W., Saunders, A., Tian, Y., Varadi, F., and Yiou, P.: Advanced spectral methods for climatic time series, Rev. Geophys., 40, 3.1-3.41, doi:10.1029/2000RG000092, 2002.

Golyandina, N., Nekrutkin, V., and Zhigljavsky, A.: Analysis of time series structure, SSA and related techniques, Chapman \& Hall/CRC, Boca Raton, London, New York, and Washington DC, 305 pp., 2001.

Hassani, H.: Singular spectrum analysis: methodology and comparison, J. Data Sci., 5, 239-257, 2007.

Idé, T.: Speeding up change-point detection using matrix compression, 2006 Workshop on Information-Based Induction Science, Osaka, Japan, October-November 2006, 31, 2006.

Idé, T. and Inoue, K.: Knowledge discovery from time-series data using nonlinear transformations, Proceedings of the Fourth Data Mining Workshop (The Japan Society for Software Science and Technology, Tokyo, 2004), Tokyo, Japan, 1-8, September 2004, ISSN 1341-870X 29, 2004.

Idé, T., and Inoue, K.: Knowledge discovery heterogeneous dynamic systems using change-point correlations, 2005 SIAM International Conference on Data Mining (SDM 05), Newport Beach, CA, USA, 571-576, April 2005.

Itoh, N., and Kurths, J.: Change-point detection of climate time series by nonparametric method, Proceedings of the World Congress on Engineering and Computer Science 2010 (WCECS 2010), San Francisco, USA, 445-448, October 2010.

Mohammad, Y. and Nishida, T.: Robust singular spectrum transform, the Twenty Second International Conference on Industrial, Engineering \& Other Applications of Applied Intelligent Systems (IEA/AIE 2009), Taiwan, 123-132, 2009.

Moskvina, V. and Zhigljavsky, A.: An algorithm based on singular spectrum analysis for change-point detection, Commun. Statist. 
Simul. Comput., 32, 319-352, 2003.

Mudelsee, M.: Ramp function regression: a tool for quantifying climate transitions, Comput. Geosci., 26, 293-307, 2000.

Nakamura, N., Kayanne, H., Iijima, H., McClanahan, T. R., Behera, S. K., and Yamagata, T.: Mode shift in the Indian Ocean climate under global warming stress, Geophys. Res. Lett., 36, L23708, doi:10.1029/2009GL040590, 2009.

Phillips, J. L.: The triangular decomposition of Hankel matrices, Math. Comput., 5, 599-602, doi:10.1090/S0025-5718-19710295553-0, 1971.

Saji, N. H., Goswami, B. N., Vinayachandran, P. N., and Yamagata, T.: A dipole mode in the tropical Indian Ocean, Nature, 401, 360363, 1999.

Scheinkman, J. A. and LeBaron, B.: Nonlinear dynamics and stock returns, J. Business, 62, 311-337, 1989.

Schreiber, T. and Schmitz, A.: Surrogate time series, Physica D, $142,346-382,2000$.
Schütz, N. and Holschneider, M.: Detection of trend changes in time series using Bayesian inference, Phys. Rev. E, 84, 21120-21128, 2011.

Theiler, J., Eubank, S., Longtin, A., Galdrikan, B., and Farmer, J. D.: Testing for nonlinearity in time series: The method of surrogate data, Physica D, 58, 77-94, 1992.

Tokunaga, T., Ikeda, D., Nakamura, K., Higuchi, T., Yoshikawa, A., Uozumi, T., Fujimoto, A., Morioka, A., Yumoto, K., and CPMN group: Onset time determination of precursory events in time series data by an extension of singular spectrum transformation, International Journal of Circuits, Syst. Signal Process., 1, 46-60, 2011.

Vautard, R., Yiou, P., and Ghil, M.: Singular-spectrum analysis: a toolkit for short, noisy chaotic signals, Physica D, 58, 95-126, 1992. 